\title{
ON THE NATURE OF THE 41-DAY CYCLE \\ OF RR LYRAE
}

\author{
L. DETRE and B.SZEIDL \\ Konkoly Observatory, Budapest, Hungary
}

\begin{abstract}
Photoelectric observations obtained with the 24-in. telescope of the Konkoly Observatory from 1950 to 1972 show that the amplitudes of the changes of the 0.57 light-curve in the course of the 41-day cycle undergo considerable cyclic variations. The length of the cycles varies between 3.8 and 4.8 yr. A new cycle always begins with a large phase-shift in the 41-day period; afterwards there is generally no further shift during one 4-yr cycle. Following Julia Balázs' hypothesis, a tentative model is suggested: The 41-day cycle is the rotation period of the star. Magnetic fields concentrated in a limited longitude zone influence the 0.57 pulsation in such a way that variations in light amplitude and phase arise as aspect effects of the 41-day rotation period. In this way, the 4-yr cycle can be interpreted as the magnetic cycle of the star. The location of the magnetic fields strongly differs in longitude from cycle to cycle causing the observed large phase shifts at the beginning of a new 4 -yr cycle.
\end{abstract}

A great number of RR Lyrae variables have strongly variable light-curves. For example, in M3 the percentage of RR Lyrae stars with light-curve variations is about 32 percent (Szeidl, 1965). The variations are especially conspicuous at maximum light and on the ascending branch where the phase-shifts can be determined very accurately. After a proper study they turn out to be periodic, with a period $P^{\prime}$, which is long compared to the main period $P . P^{\prime}$ is known at present for 26 field RR Lyrae variables; it ranges from 22.7 for $\mathrm{Z} \mathrm{CVn}$ to $138^{\mathrm{d}} 3$ for RV Cap. Among the many RR Lyrae stars in globular clusters there are only two with known $P^{\prime}$, V 30 in M53 $\left(P=0.535, P^{\prime}=37.0 ;\right.$ Wachmann, 1968$)$ and V 68 in M3 $\left(P=0.356, P^{\prime}=10\right.$ d9; Szeid1, 1965).

RR Lyrae itself has a variable light curve with periods:

$$
P=0.56683957 \text { and } P^{\prime}=40^{\mathrm{d}} 8 \text {. }
$$

We have given $O-C$ diagrams for both periods in 1970 , using the formulae

$$
C(\bar{V})=\mathrm{JD} 2414856.487+0.56683957 \times E
$$

for the visual median brightness, $\bar{V}=7.68$, on the ascending branch and

$$
C^{\prime}=\mathrm{JD} 2414905+40.8 n
$$

for the time of the most positive shifted ascending branch during the secondary period $P^{\prime}$ (Detre, 1970).

At the Budapest Variable Star Colloquium in 1968 we presented a figure based on our photoelectric observations representing the phase variation of the median brightness on the ascending branch and the variation of the visual maximum of the 0.57 light-curve in the course of the 41-day period from year to year between 1962 and 1968 (Detre, 1969, Figure 6). According to this figure the variations connected with 
the 41-day cycle were hardly perceptible in 1963 and 1967, while they were quite strong in other years. We supposed at that time that these amplitude variations were irregular. To our surprise, in the fall of 1970 and early 1971 the amplitudes were again very small. In this way a 4-yr cycle became apparent. To study the transition from the old cycle to the expected new one we observed the ascending branch and maximum of RR Lyrae as many times as possible. The results for the visual maxima and phaseshifts of the median brightness are shown in Figure 1.

At the end of the old 4-yr cycle the phase variations during the 41-day cycle died down almost completely, and the new cycle then started with a rapid increase of the

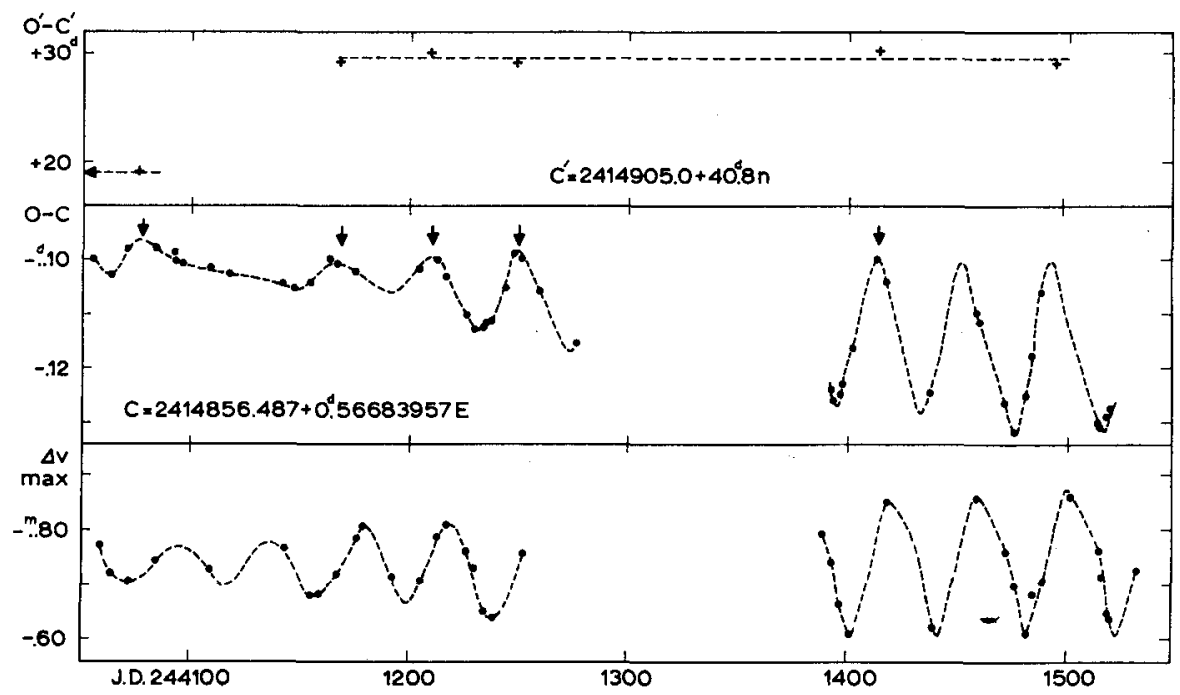

Fig. 1. The behavior of the 41-day cycle of RR Lyrae in 1971 and 1972, showing the development of a new 4-yr cycle. Below: The variation of the visual maxima of the 0:57 period. Middle: The phase-variation of the median brightness $(V=7.68)$ on the ascending branch of the visual 0.57 . light-curve. The arrows indicate the most positive shifted ascending branches. Above: The $\mathrm{O}^{\prime}-C^{\prime}$ values of the most positive shifted ascending branches calculated with $C^{\prime}=\mathrm{JD} 2414905.0+40 \mathrm{~d} 8 n$.

The new 4-yr cycle begins with a phase discontinuity of 10 days, after which $\mathrm{O}^{\prime}-C^{\prime}$ remains constant.

amplitude. The amplitude of the maximum-variations was only $0^{\mathrm{m}}, 07$ at the end of the old cycle, and then very rapidly became as large as 0.16 during 1971 and now is $0^{\mathrm{m}} .27$. Most interesting is the phase-shift in the 41-day period. $O^{\prime}-C^{\prime}$ was $+19^{\text {d }}$ throughout the old cycle (1967-1971), while for the new cycle it is $+29^{d}$, i.e. the beginning of the new cycle was accompanied by a phase shift of 10 days, about a quarter of the 41-day period.

We have performed a similar analysis of the older data. The Konkoly Observatory has about 20000 unpublished photographic observations from 1943 to 1949 and about 30000 photoelectric observations between 1950-1953 and 1956-1972. Supplemented by other photoelectric observations in 1947 (Walraven, 1949), 1953 (Hardie, 1955), 
1955 (Broglia and Masani, 1957), 1958-1959 (Cocito and Masani, 1960), 1961-1962 (Onderlička and Vetešnik, 1968), 1962-1964 (Preston et al., 1965) and by the older visual and photographic material discussed by one of us (Detre, 1943) we were able to follow the 4-yr cycle back to 1935 . The complete discussion of the entire material will be published elsewhere; here we show in Figure 2 only the amplitudes of the variation of the visual maximum and of the phase variation of the median brightness on the ascending branch of the 0.57 light-curve during the 41-day period for the years 1943-1972, together with the phase-variations connected with the 41-day period.

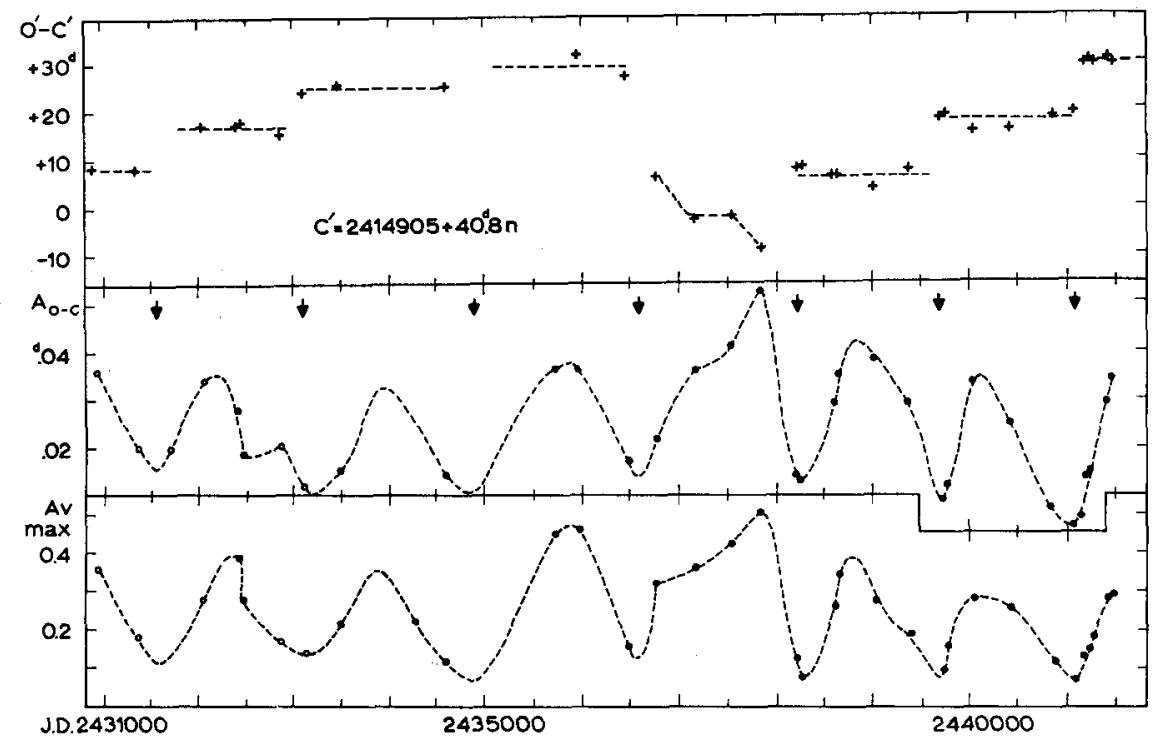

Fig. 2. Below: The amplitude of the variation of the visual maximum during the 41-day cycle vs JD. The 4-yr cycle is clearly shown. Open circles for photographic observations, approximately reduced to $V$ magnitudes, filled circles for photoelectric $V$ observations. Middle: The amplitude of the phase variation of the median brightness $(V=7.68)$ on the ascending branch of the 0.57 lightcurve during the 41-day cycle vs JD. The arrows indicate the beginnings of the different 4-yr cycles. Above: The $O^{\prime}-C^{\prime}$ values of the most positive shifted ascending branches calculated with $C^{\prime}=$ $=\mathrm{JD} 2414905.0+40 \mathrm{~d} 8 \mathrm{n}$. At the beginning of each 4-yr cycle a considerable phase discontinuity occurs, afterwards the $O^{\prime}-C^{\prime}$ values generally remain constant during one 4-yr cycle.

The amplitude variations are clearly cyclic, with cycle-lengths varying between 3.8 and $4.8 \mathrm{yr}$ and having a mean value of $4.28 \mathrm{yr}$. The longest cycles have the largest amplitudes.

The most interesting feature of the 4-yr cycle is the considerable phase discontinuity in the 41-day period at the beginning of each 4-yr cycle. After this discontinuity $O^{\prime}-C^{\prime}$ remains constant during one and the same cycle; only the strongest cycle seems to be an exception.

Our results fit in well with Julia Balázs' interpretation of the 41-day cycle proposed 13 yr ago (Balázs, 1959; Balázs-Detre, 1962; Preston, 1964). The large observed phase 
shifts in the 41-day period are hardly understandable unless the 41-day period is equal to the rotation period of the star. Magnetic fields concentrated in a limited longitude zone around the equator develop and decay over a 4-yr interval causing 41-day variations in the light amplitude of the 0.57 pulsation period and in the strength of the magnetic field as aspect effects of the 41-day rotation period of the star. The following 4-yr cycle starts with increasing magnetic fields in a different longitude, and this longitude difference between the new and old cycles gives rise to the observed phase shifts in the 41-day period. Curiously, the phase shift of 10 days, which has been observed several times corresponds to $90^{\circ}$ in longitude.

In this way, the 4-yr cycle can be interpreted as the magnetic cycle of the star. Regarding the magnetic fields, Babcock's measures (Babcock, 1955, 1958) were performed during the maximum of a very high 4-yr cycle. They show some correlation with the 41-day period (Detre, 1961), the maximum positive and maximum negative fields being associated with maximum and minimum light amplitudes respectively. Preston's measures (Preston, 1967) in 1963 coincide with the minimum of a 4-yr cycle, those in 1964 with the beginning of a weak 4-yr cycle. That might be the explanation of why he has not once found a measurable field.

\section{References}

Babcock, H. W.: 1955, Publ. Astron. Soc. Pacific 67, 70.

Babcock, H. W.: 1958, Astrophys. J. Suppl 3, 141.

Balázs, J.: 1959, Kleine Veröff. Remeis-Sternw. No.27, p26.

Balázs-Detre, J. and Detre, L.: 1962, Kleine Veröff. Remeis-Sternw. No. 34.

Broglia, P. and Masani, A.: 1957, Contr. Oss. Astr. Milano-Merate, Nuova Serie, No.105.

Cocito, G. and Masani, A.: 1960, Contr. Oss. Astr. Torino, Nuova Serie, No. 27.

Detre, L.: 1943, Mitt. Sternw. Ungar. Akad. Wiss. No.17.

Detre, L.: 1961, IAU Trans. XIB, 295.

Detre, L.: 1969, Non-Periodic Phenomena in Variable Stars, Academic Press, Budapest.

Detre, L.: 1970, Ann. Univ.-Sternw. Wien 29, No. 2, 79.

Hardie, R. H.: 1955, Astrophys. J.122, 256.

Onderlička, B. and Vetšnik, M.: 1968, Astron. Inst. Univ. Brno (Czechoslovakia), Publ. No.8.

Preston, G. W.: 1964, Ann. Rev. Astron. Astrophys. 2, 46.

Preston, G. W., Smak, J., and Paczyński, B.: 1965, Astrophys. J. Suppl. 12, 99.

Preston, G. W.: 1967, in R. C. Cameron (ed.), The Magnetic and Related Stars, Mono Book Corp., Baltimore, p. 26.

Szeid1, B.: 1965 Mitt. Sternw. Ungar. Akad. Wiss. No. 58, p74.

Wachmann, A. A.: 1968, Astr. Abh. Hamburg Sternw. Bergedorf 8, No. 114.

Walraven, Th.: 1949, Bull. Astron. Inst. Neth.11, 17. 